\title{
Learning machines. Prototipado experimental en torno al diseño, la materia y los saberes tácitos
}

\section{Ana Guerrero}

Recibido: 10.05.2021

Revisado: 10.06.2021

Publicado: 30.06.2021

Como citar este artículo

Guerrero,A., 2021. Learning machines. Prototipado experimental en torno al diseño, la materia y los saberes tácitos. Inmaterial. Diseño, Arte y Sociedad, 6 (11), pp. 110-137

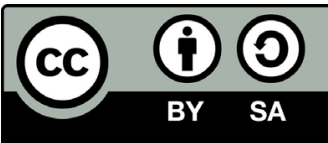




\section{Resumen}

Esta investigación tiene por objetivo evaluar y analizar cuáles son las prácticas ligadas al prototipado que nos permiten cuestionar los sistemas clásicos de producción del conocimiento, así como explorar las tensiones y oportunidades que entrelazan la experiencia con el aprendizaje directo del conocimiento tácito.

Me interesan los artefactos-dispositivos-procesos que hacen posible el cambio, la transformación y el aprendizaje del conocimiento que se está generando. Artefactos que investiguen, diseñen y cuestionen formas de producción de saberes prácticos. Prácticas sensibles al proceso de generación de conocimientos en la acción a partir de la experiencia y el diálogo con lo material.

En este caso, se parte de máquinas que se presentan como un método de investigación en el campo del diseño, como una plataforma de expresión y una herramienta inmediata que activa experiencias, donde pensar con otros no se limita a lo humano de manera performática. Máquinas con un hacer que deambula entre las lógicas de la producción digital y la artesanal, que habitan y exploran un umbral incierto de agencias mediadas por la materia.

El azar, la intuición y, a veces, la suerte, aparecen de forma inevitable en el proceso. Es necesario no tener una idea preconcebida del resultado y dar cabida a los accidentes y errores. Procesos donde la experimentación es una parte crucial, un espacio donde descubrir nuevas relaciones, posibilidades, límites y diálogos.

El prototipado de las máquinas cuestiona los paradigmas del saber, los tensa y posibilita encarnar saberes que, posteriormente, pueden ser trasladados a través del diseño como espacio, así como herramienta donde concluyen estos conocimientos.

Palabras clave: diseño, aprendizaje, experiencia, fabricación digital, artesanía 


\section{Abstract}

This research aims to investigate and question which practices linked to prototyping allow us to question the classic systems of knowledge production. To explore tensions and opportunities that intertwine experience with the direct learning of tacit knowledge.

I am interested in the artifacts-devices-processes that enable the change, transformation and learning of the knowledge that is being generated. Machines that perform acts that wander between the logics of digital and artisanal production, inhabiting and exploring an uncertain threshold of agencies mediated by matter.

In this case, starting from machines that are presented as a method of research in design, as a platform for expression and an immediate tool that activates experiences, where thinking with others is not limited to the human, in a performative way. Machines that perform acts that wander between the logics of digital and craft production, inhabiting and exploring an uncertain threshold of agencies mediated by matter.

Chance, intuition and, sometimes, luck appear inevitably in the process it is important not to have a preconceived idea of the result, as well as to allow some space for accidents and errors. Processes where experimentation forms a crucial part, a space in which to discover new relationships, possibilities, limits and dialogues. The prototyping of the machines questions the paradigms of knowledge, tenses them and makes it possible to embody knowledge that can later be transferred through design as a tool where this knowledge concludes its journey.

Keywords: design, learning, experience, digital fabrication, craftsmanship 


\section{El hacer: la práctica como investigación}

El "hacer" como poder, como acción de empoderamiento y libertad. El hacer como gesto cosmopolítico, como posibilidad de abrir fronteras, establecer diálogos y negociaciones en torno a los objetos que componen nuestro entorno, y como espacio donde repensar el famoso "saber es poder" de Focault, siendo conscientes de los otros saberes que se producen en la acción y de las verdades que se presentan al centrar las reflexiones en el prototipado.

Pablo Sztulwark defiende, en Componerse con el mundo. Modos de pensamiento proyectual (2015), que la posibilidad de transitar desde la disciplina al campo del proyecto sigue siendo una tarea necesaria. Además, debemos considerar que, cuando nos preguntamos por cuestiones que se salen de ámbitos disciplinarios determinados, estamos pensando en la problematización como construcción de relaciones nuevas y no automáticas. Sztulwark aclara que estas cuestiones están más cerca de ser aprendizajes provisorios situados en contextos determinados que certezas incuestionables fácilmente generalizadas o explicadas automáticamente.

Proyectar en diseño tiene su especificidad, puesto que es un procedimiento que construye el mundo físico y material donde se despliega la vida.

Según Sztulwark, pensar críticamente implica producir nuevas preguntas capaces de forjar nuevas relaciones entre los saberes, lo cual no significa, en los campos creativos y prácticos como los de la arquitectura y el diseño, desdeñar aquellos saberes que conforman nuestra plataforma de conocimiento. Si pensar es hacer de cada cosa un problema, y si problematizar es poner en cuestión la potencia de un saber frente a un problema nuevo, nos podríamos preguntar si el saber es simplemente una construcción de la realidad y, por tanto, una mirada sobre el mundo de lo real.

Históricamente, el conocimiento se ha dividido en dos grandes grupos: el conocimiento explícito y el conocimiento tácito.

Esta investigación pone el foco en el segundo tipo de conocimiento, el tácito. Se trata de un concepto creado por el científico y filósofo Michael Polanyi (18911976) en el libro Knowing and Being (1969). Polanyi definió el conocimiento tácito como "aquello que sabemos y que no puede ser comunicado completamente por medio de palabras", entendiendo el "sabemos" como una habilidad o capacidad de desempeño y no como un simple dato o una información que tenemos en la mente. 
El tácito es un conocimiento que está estrechamente vinculado a la experiencia individual; implica crear y reconocer creencias personales, perspectivas y posicionamientos. Por eso es poco visible y mucho más difícil de transmitir por medios tradicionales que el explícito.

Se desarrolla desde la experiencia directa y la acción, y es altamente pragmático. Pasa por lo vivencial, lo corporal, y posee una gran especificidad, ya que únicamente lo poseen los actores del contexto donde se produce. De Souza defendía que es imposible su acceso sin la interacción directa y personal con los actores que lo poseen. $\mathrm{Su}$ transferencia depende principalmente del esfuerzo creativo para expresarlo.

"La principal característica de la transmisión del conocimiento tácito es que su acceso es imposible sin la interacción directa y personal con los actores que lo poseen, pues su transferencia depende principalmente del esfuerzo creativo para expresarlo a través de imágenes verbales, metáforas, símbolos heurísticos y analogías”. (De Souza, 2000).

Brown y Duguid (1998) consideran que parte del conocimiento se produce y mantiene colectivamente. Estos autores defienden que el conocimiento se genera fácilmente cuando se trabaja conjuntamente, siendo este fenómeno denominado comunidad de prácticas. A través de la práctica, una comunidad desarrolla una comprensión compartida sobre lo que hace, cómo lo hace y cómo se relaciona con las prácticas de otras comunidades de práctica. De esta forma el know how (la dimensión técnica del conocimiento) comprende la habilidad para poner en práctica el know that. Por otra parte, el know how es importante a la hora de convertir el conocimiento en operativo.

\section{Artesanía y fabricación digital: ¿opuestos?}

Los aparatos son dispositivos de destino que hacen época. Un mismo arte puede ser maquinizado de manera muy diferente. La invención de los aparatos procede del desajuste y la diferenciación, que tiene el sentido de una necesaria emancipación. Lo que implica que las únicas verdaderas revoluciones no son políticas, sino culturales. La invención de un medio de comunicación es una cosa, la de un aparato, otra distinta. Una revolución cultural es el surgimiento de un aparato (Déotte, 2013).

La tradición de los oficios vinculados a la artesanía está adquiriendo cada vez mayor valor y estima en un momento en el que avanzar parecía ir solo de la mano de un mundo completamente mediado por lo tecnológico. Hace tiempo que tenemos 
la idea de que la artesanía y la tecnología habitan en mundos casi opuestos. Ambas responden a conceptos muy distintos, y todos tenemos en mente ideas que rápidamente se asocian a ambas. Mientras que la artesanía evoca el sabor de épocas pasadas, del trabajo bien hecho, de técnicas que sobreviven gracias al trabajo de unos pocos donde la pericia manual prevalece, la tecnología nos sugiere progreso, vértigo, modernidad y también estandarización. En mi opinión, no tienen que estar necesariamente separadas, sino que debemos saber cuándo utilizar cada una según el objetivo al que queramos llegar o el tipo de trabajo que estemos llevando a cabo. Al fin y al cabo, muchas de las tecnologías parten de la imitación de los procesos manuales para facilitarlos u optimizarlos.

Considero que un posible camino hacia las prácticas futuras en el ámbito del diseño y de otras disciplinas creativas, puede ir orientado hacia una hibridación entre "mano y máquina”, que aceptaría la tecnología como aliada y no como enemiga. Algunas disciplinas en las que la tradición de lo manual perdura ven en las nuevas tecnologías una competencia desleal, pues competir con los ritmos acelerados en los que está sumergida la industria es inviable y difícilmente alcanzable. Esto se traduce de forma directa en el precio de lo que se produce, quizá por eso acudimos a un Ikea antes que a un carpintero de barrio si queremos un mueble.

Pero la tecnología puede ser algo más, una herramienta de extensión de nuestro propio cuerpo y saber: impresiones 3D que se adaptan a cada individuo, fresadoras (CNC) con acabados manuales o prototipos hechos con una cortadora láser sobre los que pensar posteriormente.

\section{Haceres manuales}

En su libro El Artesano (2008), Richard Sennett explica la relación entre las herramientas y las máquinas, así como el desarrollo de las habilidades que requiere el artesano y su actitud ética. Toda artesanía se fundamenta en una habilidad desarrollada en alto grado. Como decía Pallasma, (2014), la repetición conforma la experiencia. La recompensa emocional que ofrece la artesanía con el logro de conseguir una habilidad es doble: el artesano se basa en la realidad tangible y puede sentirse orgulloso de su trabajo. Sennett establece dos argumentos básicos acerca de la interacción entre las acciones físicas de la mano y la imaginación.

Sennett aborda todas estas prácticas como si se trataran de laboratorios en los que es posible investigar sentimientos e ideas. El artesano necesita desarrollar relaciones específicas entre el pensamiento y la creación, entre la idea y la ejecución, la 
acción y la materia, el aprendizaje y la ejecución, la identidad propia y la obra, y entre el orgullo y la humildad, e incorporar la herramienta e interiorizar la naturaleza del material.

Uno de los aspectos de la lógica de la transmisión que plantea la artesanía es el de pasar el conocimiento de generación en generación. Los llamados "métodos de caja negra" recogían todas esas actualizaciones metodológicas en las que el diseñador-artesano no pretendía establecer el control estricto sobre los motivos y sus decisiones proyectuales. Los métodos tradicionales de la artesanía son de este tipo: el artesano sabe lo que hace pero tiene grandes dificultades para mostrarlo, explicarlo y transmitirlo paso a paso. Las dificultades se agravan si pretendemos que expliquen las profundas relaciones de causa-efecto que se dan entre la información disponible y los resultados de sus decisiones formales.

El dispositivo que se plantea, entonces, debería tener como objetivo construir materiales para pensar, para crear y, por qué no, para inventar.

\section{El taller como espacio de aprendizaje}

Sztulwark defiende que la enseñanza de nuestra disciplina se desarrolla en los talleres, que es un trabajo de taller. Esa configuración espacial y de usos que representa este ámbito forma parte de una cierta naturalización de la tarea de la enseñanza de la arquitectura y del diseño que habría que replantear.

Si consideramos el espacio del taller en relación con el proceso de creación, podemos ver que la idea misma del taller remite a un proyecto dentro del cual el pensar se asocia a una materialización. Si el taller se toma solo como ámbito de configuración espacial dentro del cual desarrollar una cierta práctica individual o grupal, pero no como un espacio de pensamiento colectivo, nos encontramos con una práctica desnaturalizada. Por eso interrogar el espacio del taller supone considerar lo que significa pensar con otros.

Los artistas y artesanos que trabajaban en las logias (comunidades de trabajo desarrolladas en los siglos XII y XIII que trabajaban en la construcción de una gran iglesia, generalmente una catedral), adquirieron la cualificación necesaria aprendiendo de un maestro y realizando prácticas sobre el terreno según el principio de imitación (enseñar-copiar). Las reglas del gremio coartaban en gran medida la libertad de expresión individual. 
Los talleres artesanales y sus maestros conviven ahora con la existencia de los talleres de fabricación digital, más comúnmente conocidos como fab labs, espacios de innovación, creación y experimentación, muy vinculados a la sociedad y sus cambios emergentes. Estos espacios fueron impulsados por el MIT (Massachussets Institute of Technology) en el año 2000.

\section{Haceres digitales}

El término fabricación digital surgió hace años y, aunque el propio nombre lo define -la digitalización de los procesos de fabricación-, son muchos los factores que intervienen, desde el entendimiento más técnico hasta las redes y comunidades que se generan en sus espacios y la forma de compartir conocimiento.

En su artículo Fab Labs y makers: cultivar otras subjetividades (2019), José Pérez de Lama propone un listado de las razones más comunes y destacadas por las que algunas personas entran a formar parte de la comunidad de makers, que catalizan estos «nuevos» laboratorios de creación.

Una de las principales razones sería la del placer de hacer, la posibilidad de hacer cosas físicas y, relacionado con esto, la posibilidad de hacerlo por nosotros mismos dentro del movimiento Do It Yourself, que aparece en la década de 1960-70, o Do It With Others, que contrasta con la dependencia de los sistemas globales de producción, los cuales nos resultan complejos de comprender y acceder. De esta forma actuamos de agencias en la construcción de nuestro propio mundo, que podemos expresar e incluso inventar ${ }^{1}$.

Esto genera un sentimiento activo en la interpretación y la construcción del mundo contemporáneo. Además, esto se produce, no como individuos aislados, sino como parte de una comunidad local, de cada fab lab o grupo de makers, y una de una comunidad global. Otras de las razones que se apuntan es el interés por aprender en espacios y de maneras diferentes de las habituales.

La posibilidad de habitar espacios en los que prima el intercambio de conocimientos, abre grandes posibilidades y oportunidades de aprender, compartir, hacer y

\footnotetext{
${ }^{1}$ Whole Earth Catalog (1968-1972). Fue un fanzine contracultural que ofrecía herramientas y sugerencias para optimizar la vida cotidiana. Se considera un precursor en papel de los buscadores de internet como Google, o incluso de internet mismo, por su intención de poner al alcance del lector «todo» el conocimiento generado por la humanidad. Entre la información que se podía encontrar en él había resúmenes de textos científicos, guías de estilo de vida, recetas de cocina vegetariana, artículos sobre misticismo hindú, budista y New Age, tutoriales de DIY, consejos para la protección del medio ambiente, etc.
} 
decidir acerca de nuestro efecto sobre el entorno y cómo éste nos afecta para transformarlo pensando en el bien común.

\section{Haceres que convergen}

El término artesano ha evolucionado con los años hasta lo que ahora se conoce como «artesano digital»o «artesano postdigital». Muchos artesanos reniegan de estas tecnologías (por desconocimiento o tradición), y vinculan la artesanía a procesos manuales y, a través de ello, al desarrollo de habilidades en la construcción y manufactura de objetos. Pero lo cierto es que muchos otros se han sumado al movimiento y comparten procesos creativos y productivos con otras disciplinas como, por ejemplo, el diseño, e incorporan al saber hacer manual las ventajas de las nuevas tecnologías.

"Partisans for craft - I count myself among them - may be tempted to simply celebrate the relevance of hand skill in the context of innovation. We are relieved (possibly even pleasantly surprised) to find that artisanal know-how still has a role to play in innovation. Yet this is not going far enough. We should not simply see craft as the static ground from which the digital emerges. Instead, we should understand the interaction between the analogue and the digital as recursive, with each array of techniques decisively inflecting the other." (Adamson, 2015)

Noam Dover 2, en su proyecto Embracing the digital to the handmade (2017), une la tecnología digital con métodos de elaboración de moldes de soplado de vidrio. El proyecto trata sobre el potencial del vidrio soplado en molde y su capacidad para contar la historia de su propio proceso de fabricación. Incorpora la fabricación digital en el proceso de creación de los moldes para generar innovaciones y nuevos conocimientos en la artesanía produciendo nuevas técnicas y culturas materiales.

"Los artesanos habían inventado el torno de alfarería; ellos crearon la cerámica, las ollas y los metales y vidrio fundidos y refinados; fabricaron tubos de metal para soplar el vidrio. El enfoque innovador de la fabricación de herramientas es muy relevante para la forma en que percibo que nosotros, los artesanos, podemos innovar y aplicar nuevas tecnologías a nuestro oficio". (Dover, 2017). 


\section{Prototipar como gesto cosmopolítico}

El diseño cosmopolítico adopta la lógica de lo infradeterminado, una transformación localizada y continua capaz de crear las condiciones para la negociación y la colaboración entre diversas entidades. Las alteraciones que se producen con este diseño hacen visible la variedad de posibilidades contenidas de la realidad.

El diseño siempre es codiseño, los materiales diseñan, tienen una acción. Cualquier proyecto es participativo, más allá de lo humano. Prototipar es, por lo tanto, una acción performativa continua, una acción que vuelve repetidamente a transformar un mismo objeto para buscar otras maneras de negociación con los usuarios o aquellas entidades a las que el objeto se destina. Es una transformación localizada y continua, capaz de crear condiciones para la negociación y la colaboración, pero no podemos diseñarlo todo. Cualquier acción nuestra se compone de una serie de acciones de otras entidades. Prototipamos con materia, tecnología, otros humanos, otros no humanos... todos, en mayor o menor medida, participan de la acción.

Actualmente la tecnología desempeña un papel esencial en todo lo que producimos y pensamos. Tendemos a pensar que la tecnología es neutral (Winner), pero en su diseño ya lleva inscrita una teoría política. Bruno Latour decía que para cada programa hay un contraprograma; las tecnologías sirven como herramientas políticas porque delegan en el material y traducen una necesidad social en individual.

\section{Cosas que hablan}

En el texto The prototype: more than many and less than one (año), Alberto Corsín reclama la creación de prototipos como un discurso cultural actual y los analiza como figuras de suspensión y expectativa. Coloca el prototipo en una posición privilegiada desde donde examinar algunos debates clave en la teoría social actual, que se alimentan de los debates más recientes sobre la materialidad del proceso político. Para Corsín, el prototipo funciona como descriptor, tanto para un objeto epistémico como para una cultura epidémica. Es un lenguaje y una referencia para una nueva conciencia tecnopolítica de oficio, habilidad y autoorganización comunitaria, y explica que las cualidades experimentales y abiertas 
de los prototipos se han convertido en un sustituto de nuevas experiencias culturales y procesos de democratización. Son cosas que hablan y generan un debate sobre las cualidades objetivas y materiales de la cultura. También sitúa el «prototipo» actual como una «trampa» para una figura contemporánea de posibilidad y expectativa. El trabajo de esa trampa sería mantener la socialidad en suspensión.

Los objetos que se presentan en esta investigación son, por lo tanto, artefactos performáticos que muestran cualidades experimentales y de coproducción con otros. Es imprescindible entender estos dispositivos como prototipos, y volver a ellos una vez puestos en marcha. Observar la reacción humana y matérica y volver a empezar, transformando la pregunta y evolucionando de forma conjunta.

\section{La máquina como concepto complejo}

Maurizio Lazzarato en el texto La máquina (2006) interpreta el punto de vista de Deleuze y Guattari, y afirma que el capitalismo no es un «modo de producción», ya no es un sistema, sino un conjunto de dispositivos de servidumbre maquínica y, a la vez, un conjunto de dispositivos de sujeción social. Afirma que los dispositivos son máquinas y remarca, siguiendo a Deleuze y Guattari, que las máquinas ya no dependen de la techne sino que la máquina tecnológica es solo un caso de maquinismo. Defiende que hay máquinas técnicas, estéticas, económicas, sociales, etc., en las que las funciones, los órganos y las fuerzas del hombre se agencian con ciertas funciones, órganos y fuerzas de la máquina técnica; juntos constituyen un agenciamiento.

La máquina no es solamente la totalidad de sus piezas, los elementos que la componen: "es portadora de un factor de autoorganización, de feedback y de autorreferencialidad incluso en su estado maquínico" (Bryan, 2014). Tiene un poder, el poder de abrir procesos de creación. De este modo, por extraño que pueda parecer para la tradición del pensamiento occidental, la «subjetividad» se encuentra a la vez del lado del sujeto y del lado del objeto.

Las maquinarias de servidumbre y de subjetivación trabajan sobre las relaciones. Su acción, según la definición de poder de Foucault, es una acción sobre una acción posible, una acción sobre individuos «libres», 
que siempre pueden actuar de forma diferente. Esto no implica solamente fracasos eventuales en la sujeción, resultados imprevisibles, la activación de desviaciones, de trucos, de resistencias de los individuos, sino también la posibilidad de procesos de subjetivación independientes, autónomos. Encontramos aquí el concepto de: «máquina abstracta».

La razón ontológica de la máquina es hacer, no ser.

Una máquina es todo aquello que produce un conjunto de operaciones y efectos, estos efectos pueden ser de naturaleza tan diversa como todas las entradas que la atraviesan en diferentes contextos históricos y culturales. Por ejemplo, Levi R. Bryant afirma que las grandes obras de arte son máquinas porque resuenan, porque son capaces de producir nuevas manifestaciones locales como resultado de las otras entidades existentes. De alguna manera manejan el medio cultural e histórico en el que «caen» o aparecen, produciendo algo nuevo con el resultado. Divide las máquinas en poderes y manifestaciones: los «poderes» son las características de esa máquina, independientemente de si alguien los conoce, los ha observado o si la máquina está activa o no, mientras que las manifestaciones son el producto de la operación de un poder en una entrada particular.

Frente a una máquina, nuestro primer pensamiento no se centra en sus propiedades o cualidades, sino en sus operaciones. La máquina es algo que funciona. En su libro "Onto-cartography. An ontology of Machines and Media” (2014), Levi R. Bryant agrega a la definición de máquina la necesidad de la producción no solo de una entrada, sino también de una salida. Las entradas realizan una transformación a través de una operación y pueden originarse desde fuera de la máquina o desde dentro. Por ejemplo, una entrada puede originarse dentro de nuestro cuerpo.

\section{Las máquinas: hacia una investigación tácita en el diseño}

Las máquinas se presentan como un método de investigación en el diseño, como una plataforma de expresión y una herramienta inmediata que activa experiencias mediando entre lo humano y lo no humano de manera performática. De forma intuitiva, genero máquinas o sistemas mecanizados que permiten generar un umbral de incertidumbre. 
La máquina como camino, que pasa por lo material pero en la que el peso o el interés mayor no recae en el resultado, sino en el proceso y en la máquina que lo produce. Más que una investigación que pone el foco en la experimentación material, podría decirse que es una experimentación procesual.

"Si aceptamos como altamente improbable la aparición de un control total del proceso, también tendríamos que aceptar como muy improbable la existencia de lo que sería un método único" (Martí Font, 1999. p. 150)

La máquina tiene que hacer una cosa distinta de lo que ya sé, de lo que ya pienso, de lo que ya siento. Ha de tener algo incomprensible, incontrolable para mí. Lo decisivo, desde el punto de vista de la experiencia, no es de qué máquina se trate, sino de lo que nos pasa cuando la utilizamos. Una persona que utilice la máquina y no note nada, no vea esa realidad de otro modo, no se cuestione su uso, estructura o estética o no le genere nuevas preguntas, será una persona que no haya tenido una experiencia.

Levi R. Bryant desarrolla, a partir de la obra de Delueze y Guattari, la teoría de que las máquinas son binarias, que pueden acoplarse a otras máquinas que proporcionan un flujo; esta segunda máquina funciona como un medio. La consecuencia de esto es que las máquinas, al realizar operaciones en los flujos o las entradas, tendrán que lidiar con los poderes que caracterizan estos flujos. Es por esta razón que las máquinas no son soberanas de los flujos que las atraviesan. [...] En otras palabras, hay muchos casos en los que las máquinas que fluyen a través de una máquina modifican la máquina. (Bryant, 2014)

En las máquinas que propongo, el cuerpo interviene en todas ellas como medio, como la segunda máquina que permite que se desencadene el proceso. Y aunque siempre se necesite al ser humano para iniciarla, ya sea pulsando un botón que active un mecanismo o girando una manivela, estas máquinas requieren de una implicación manual y corporal completa. Este tipo de máquinas me interesan porque exigen humanidad. 
¿Hasta dónde se extiende el límite de las máquinas?

¿Qué producción de estéticas y conocimientos se generan?

¿Qué realiza la máquina y cómo dialoga con el contexto?

Las máquinas utilizan técnicas que no podemos dominar por completo. La coincidencia y la intuición desempeñan un papel imprescindible en el uso de la propia máquina y en los objetos resultantes.

El azar, la intuición y, a veces, la suerte aparecen de forma inevitable en toda la investigación; es necesario no tener una idea preconcebida del resultado y dar cabida a los accidentes y errores. Es así como han surgido realmente la mayoría de las experimentaciones, mediante derivaciones de "fracasos" anteriores, como nuevas opciones y oportunidades que toman forma confiando en las sensaciones y la propia intuición. El camino entre la máquina y el objeto que produce es siempre inseguro y discontinuo, pues todas las máquinas poseen un componente de aleatoriedad. ¿Cómo afecta la aleatoriedad y el cuerpo a las estéticas y a los aprendizajes? ¿Hasta dónde puedo intervenir o controlar el proceso? Esto es lo que las hace interesantes, el hecho de no saber qué esperar.

La toma de decisiones formales de las máquinas nunca es neutra, siempre responde a una serie de características y premisas que han de cumplir. En primera instancia, han de ser accesibles materialmente, cuanto más fácil de conseguir sean los materiales de las que están hechas, más fácil resultará que otra persona se aventure a replicarlas, a modificarlas o a comenzar un proceso semejante con otras máquinas.

Todas ellas responden también a un ejercicio de simplificación de otra versión anterior sobre la que se basan y sobre la que se han modificado en mayor o menor medida. Para que esto funcione es necesario un proceso de síntesis, análisis y entendimiento de la propia máquina, que pasa por prototiparla. Aunque las máquinas constan de piezas sencillas de manufacturar, me he servido de las herramientas de fabricación digital para construirlas, sobre todo de la fresadora CNC para cortar la madera, que es el material de base de todas mis máquinas.

Como diseñadora, asumo el papel de intérprete entre la necesidad de responder a la pregunta de esta investigación (iqué prácticas ligadas al prototipado nos permiten cuestionar los sistemas clásicos de produc- 
ción del conocimiento?), y las máquinas como resultado de un proceso experimental que tienen como objetivo responder a esta pregunta $\mathrm{o}$, más bien, abrir el debate y entablar un diálogo entre las disciplinas que nos ocupan y sus modelos de producción. Esta práctica pasa por mi saber hacer, con toda la sensibilidad estética y técnica que eso conlleva. Si bien es cierto que, como ya hemos visto anteriormente, la experiencia y el conocimiento tácito que se produce en la acción es muy compleja de transmitir y que esta investigación se basa en mi experiencia particular prototipando estos objetos, intentaré asumir el reto implícito de transmitir con palabras e imágenes el camino que ha construido este proceso. Quisiera advertir al lector de que este texto no pretende en ningún momento funcionar como una suerte de manual o de instrucciones a seguir, y que espero que esta investigación pueda motivar el comienzo de (otros) procesos propios.

\section{Comenzar a maquinar}

Una de las premisas con las que se origina la búsqueda es la de poner a prueba la copia como método de aprendizaje radical. Copiar para recrear, rehacer y repensar. La copia de una máquina como forma de apropiación o uso y transformación para generar otras maneras de experimentación tácita. Copiar máquinas de forma radical ya es aprender. Observar y reproducir para entender y experimentar lo que esconden. Para ello, es necesaria una alta capacidad de autoaprendizaje y adaptabilidad, así como una acción sensible y cuidadosa.

Mi investigación comienza con una experimentación material, donde lo procesual y lo experimental son centrales y buscan límites formales con los que explorar e hibridar conocimientos. Durante esta exploración, y con la correspondiente atención a la documentación (no necesariamente escrita), se generan nuevos descubrimientos, nuevos conocimientos.

\section{"Visualizing Gravity»}

Este ejercicio consistió en localizar un referente interesante e intentar aproximarme a su trabajo recreando de cero su proyecto (en este caso, sus máquinas). De esta forma, podía valorar los conocimientos y aprendizajes que se suceden, y que no podrían darse sin hacer este ges- 
to de réplica. Para este reto me fijé en el proyecto Less CPP, Catenary Pottery Printer, una máquina para la fabricación de alfarería a partir de la curva catenaria. Este proyecto se centra, más que en el resultado, en los esfuerzos del proceso, que se hace posible gracias a la fabricación de esta máquina.

Esta máquina lanza un nuevo concepto «más paramétrico, menos digital», ya que permite manufacturar piezas de diseño paramétrico ${ }^{2} \sin$ usar ordenadores, programas de diseño digital o laboratorios de fabricación digital. Los objetos resultantes de este proceso-máquina abren diálogos que van desde la generación de conocimiento en los contextos académicos y científicos hasta la producción de piezas de diseño en las comunidades de artesanos.

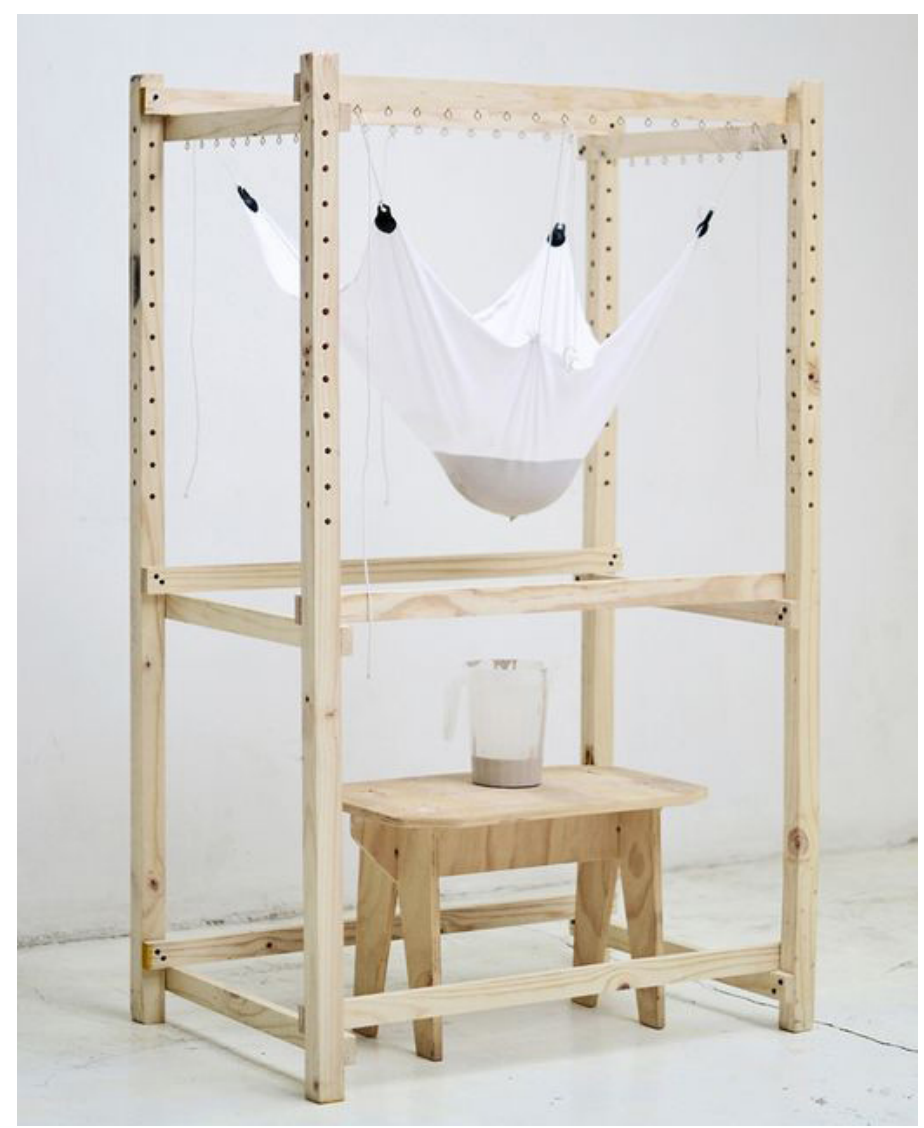

Figura 1. Proyecto Less CPP, Catenery Pottery Printer (2014)

\footnotetext{
${ }^{2}$ El diseño paramétrico es una técnica avanzada de diseño digital que permite introducir una serie de variables o parámetros, tales como límites espaciales, volúmenes o temperaturas, en un software especializado para manipularlos mediante algoritmos y obtener así diseños geométricos más complejos, versátiles y originales.
} 
El objetivo principal de esta máquina era desarrollar una investigación orientada al diseño de productos a través de una máquina (muy) low-tech que a su vez dialoga con las nuevas lógicas de producción de la fabricación digital. La máquina debía autofabricarse y ser capaz de visibilizar la energía con la que trabaja (en este caso, la gravedad), en un proceso de combinación del conocimiento en el paso de lo explícito a lo tácito, en un proceso de internalización.

En el proyecto se utiliza un tipo de tecnología maquínica como vínculo directo con la artesanía y se aprovecha la oportunidad de generar más artefactos autofabricados. Asimismo, se explora el tipo de agencias que se generan entre el artesano y la tecnología, la materia y la tecnología y, por último, entre el usuario y la propia materia, a fin de observar qué tipo de desplazamientos disciplinares y qué otras preguntas atraviesan la investigación, y qué conocimientos se generan en la acción de esta combinación.

El proyecto se centra en el proceso de internalización (aprender haciendo). Es un proceso de incorporación del conocimiento explícito en conocimiento tácito, que analiza las experiencias adquiridas en la puesta en práctica de los nuevos conocimientos, y que se incorpora en las bases del conocimiento tácito de los miembros de la organización, en forma de modelos mentales compartidos o prácticas de trabajo.

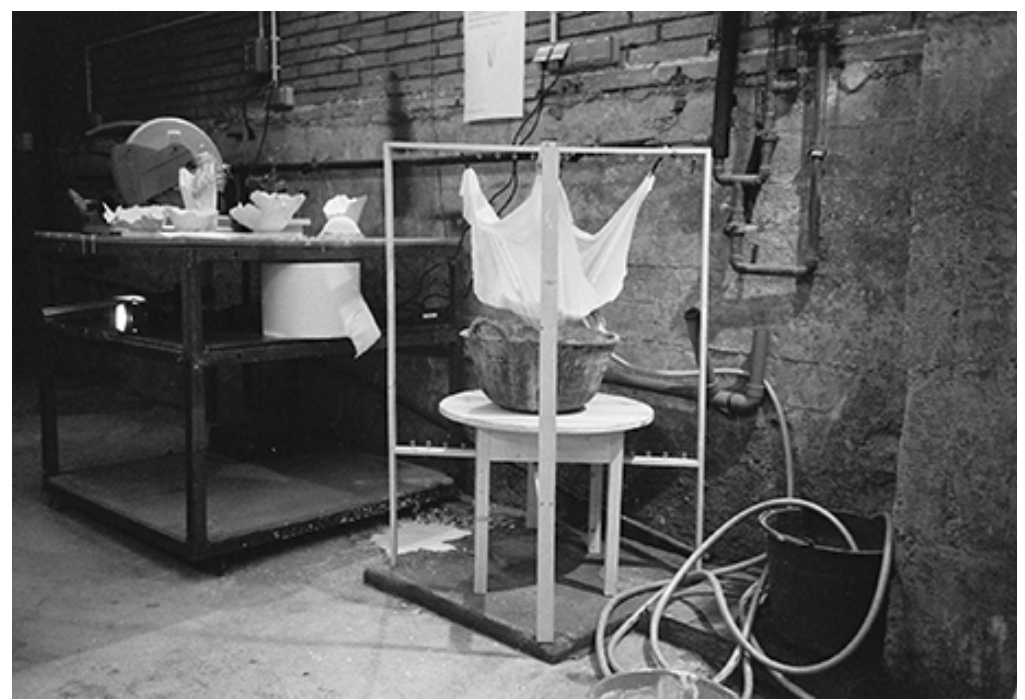

Figura 2. Máquina Visualizing Gravity.

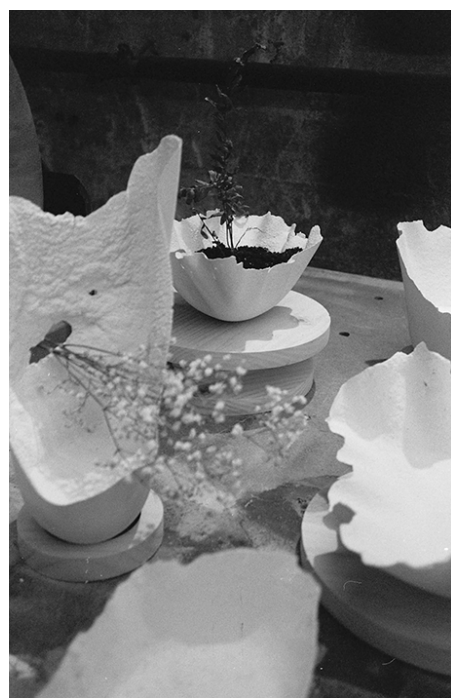

Figura 3. Resultados de la máquina Visualizing Gravity. 


\section{"La máquina de la bolita"}

La segunda máquina de la cual parto es la Kinetic drawing machine for station to station de Olafur Eliasson. La máquina forma parte del proyecto «estación a estación» de Aitken.

Se trata de una máquina de dibujo dinámica instalada en el vagón de un tren, que utiliza el movimiento del tren para mover una bola de tinta por la superficie circular de una hoja de papel blanca. A medida que la esfera pigmentada de negro se desliza, rebota y rueda sobre la capa de papel, va trazando la topografía que encuentra el tren a su paso. El resultado es una representación visual de las ciudades y pueblos que atraviesa. El ejercicio se repitió 20 veces hasta volver a Berlín, la estación de origen.

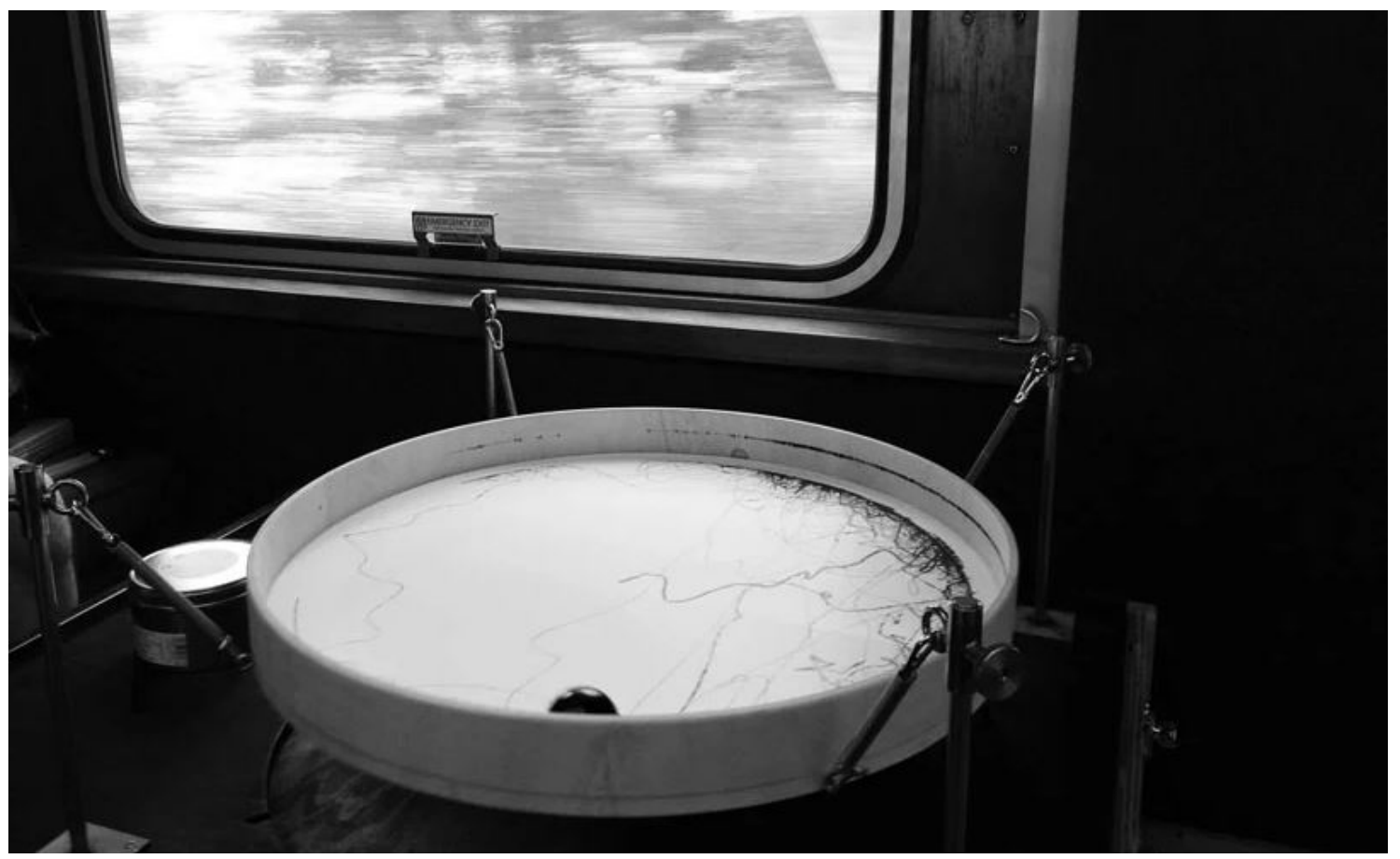

Figura 4. Proyecto Kinetic drawing machine for station to station de Olafur Eliasson (2013) 
La primera acción tiene que ver con entender el funcionamiento de la máquina. Al no poder acceder a su planimetría ni a versiones anteriores del dispositivo, lo único que sabemos es que funciona tal y como nos la muestran. No sabemos si es el mejor modo de utilizarla, si con otros materiales funcionaria mejor o si las dimensiones establecidas cumplen un aspecto funcional o puramente estético, entre otras cuestiones de la larga lista de incertidumbres y especulaciones. Por lo que hay que probar.

Para entender el funcionamiento y la lógica de la máquina generé un primer prototipo muy básico y algo precario con una quesera, una bola de poliespán empapada en tinta y folios de $120 \mathrm{~g}$. El dispositivo funcionaba, pero faltaba el componente de movilidad. Del tren pasamos a la moto, mi vehículo habitual, y tuve que repensar la estructura original para adaptarla al nuevo transporte.

Esta máquina, apodada cariñosamente «la máquina de la bolita», funciona originariamente con $2 \mathrm{D}$, papel y tinta. Para aproximarme un poco más al lenguaje de la fabricación digital y a sus resultados, decidí corromper la máquina implementando la lógica de la sustracción de material de una fresadora, pasando del 2D al 3D. Ello derivó en una nueva experimentación material.

Realicé muchas pruebas con yeso, cemento, barro, etc., pero con ninguno obtuve un resultado demasiado interesante. El estado en el que ha de estar el material para funcionar es muy concreto. La capa superior que roza la bola ha de estar seca para que pueda rodar y no se levante al moverse, pero la parte inferior ha de seguir húmeda para que la bolita sea capaz de marcar el recorrido. A pesar de haber cambiado el lenguaje del objeto y haber intentado pasar del 2D al 3D, seguía quedándose en un estado bastante plano.

Después de probar con todos estos materiales y varios tipos de bolas (de corcho, plomo, poliespán, petanca, ping-pong y goma), la máquina quedó descartada para la búsqueda de otros lenguajes. 


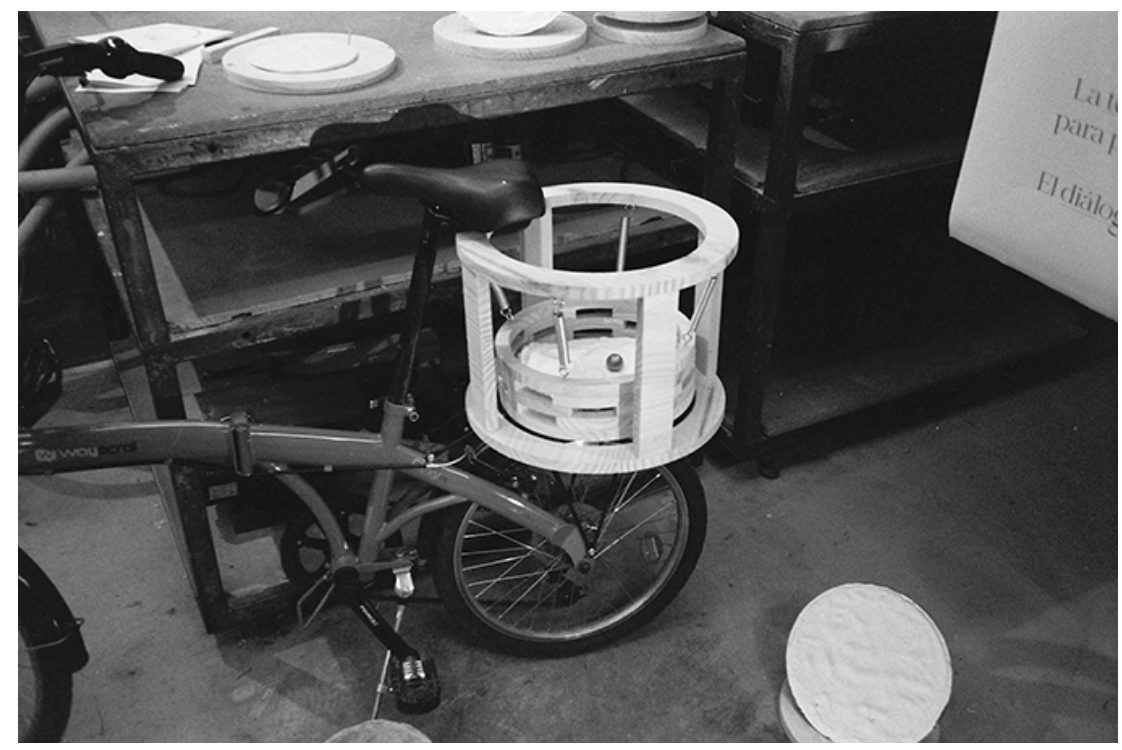

Figura 5. Máquina de "la bolita"

\section{Hackear el molde}

A partir de la fusión de dos de las técnicas utilizadas en las experimentaciones «fallidas»: la abrasión de plástico con acetona o disolvente y la prueba del poliespán y la acetona en la «máquina de la bolita», entendí que las máquinas no tienen por qué producir por sí mismas un objeto «final», sino que pueden utilizarse para generar de forma aleatoria el molde del objeto.

En primer lugar, utilicé el poliespán agujereado de la primera prueba descartada, lo llené de cemento y lo usé de contramolde, tras crear un encofrado de cinta para que no se derramara. Al secarse el cemento, vi que la pieza era muy delicada, pues los surcos que genera la acetona pueden llegar a ser muy pequeños e impredecibles y, en lugar de retirar el molde de forma manual, lo deshice por completo añadiendo más disolvente. 


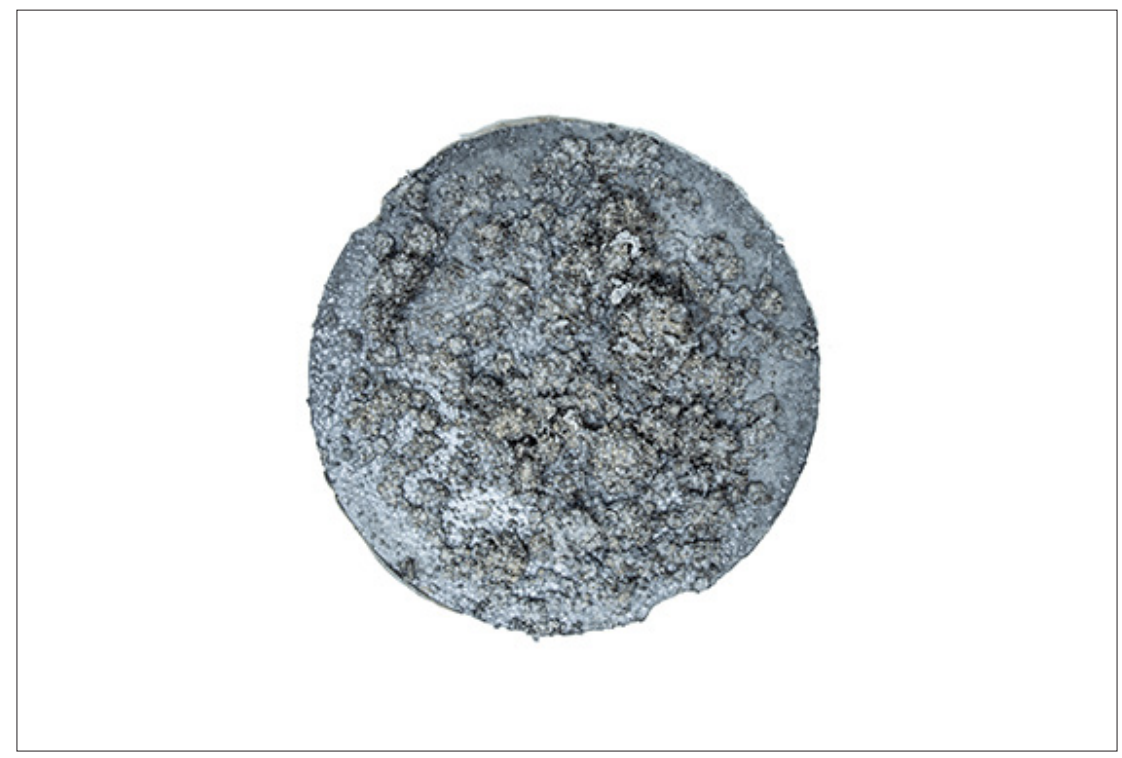

Figura 6. Prueba de poliespán y acetona en plano

El resultado fue sorprendente, pues las bolitas de aire del poliespán generan formas y surcos que, al verter el cemento, se solidifican creando algo que parece una especie de alga fosilizada.

$\mathrm{Al}$ evaluar las posibilidades de la unión de estas técnicas, decidí mecanizar el proceso (hasta ahora manual), construyendo una nueva máquina que reforzara el componente del azar en la producción del molde, que por definición debía generar objetos iguales.

Es aquí donde aparece la máquina de rotomoldeo manual. Normalmente, la función principal de este tipo de tecnología es generar objetos de cerámica, o algún material semejante, y líquido, que al girar se adhiere a las paredes de un molde previamente colocado con la forma deseada para obtener réplicas de figuras iguales y huecas.

En mi caso, lo que se genera en la máquina es el propio molde tras colocar en el interior una pieza de poliespán (una esfera en la mayoría de las pruebas, por las posibilidades que ofrece esta forma al modificarse $360^{\circ}$ ) y añadir disolvente por un orificio. 


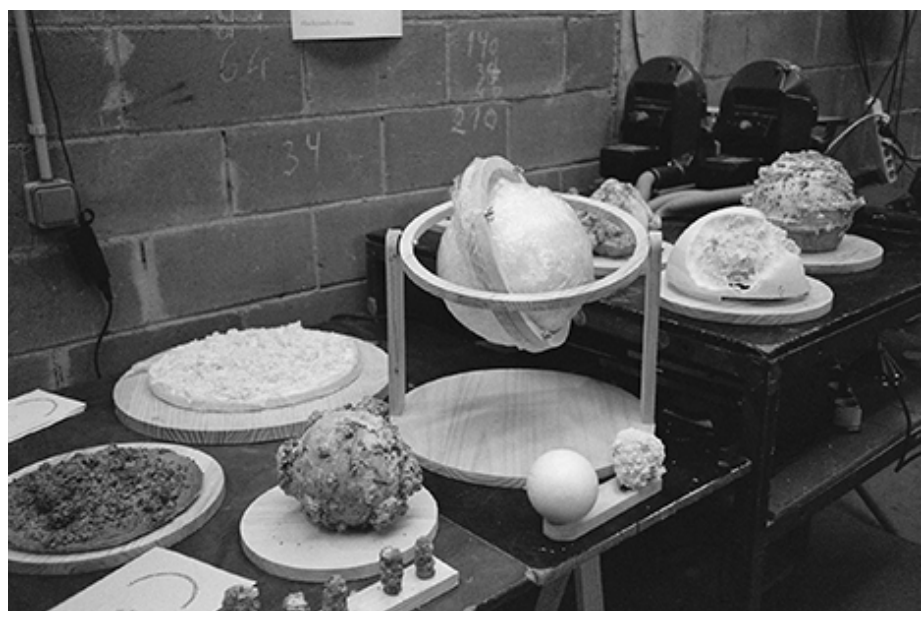

Figura 7. Máquina hackeando el molde.

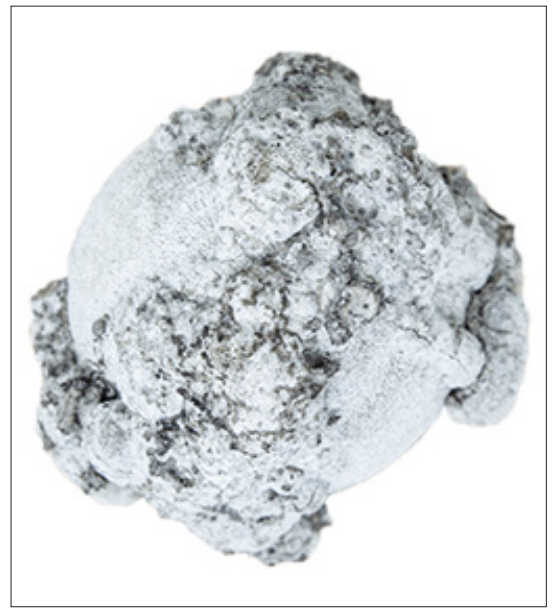

Figura 8. Resultado de la máquina hackeando el molde.

\section{Teachable Machine}

En Material Utopias (2017), Amanda du Preez hace una reflexión en torno a la desmaterialización y propone entenderla, en lugar de como una negación o una despedida de la materialidad, como un replanteamiento extenso y fundamental de la multiplicidad de la materialidad más allá de su conexión con la entidad del objeto. [...] A diferencia de la idea de que la desmaterialización implica una estética según la cual lo conceptual es superior o sobredimensiona la materialidad, Preez interpreta la desmaterialización como una estética en la que lo conceptual ya es siempre material. Esta estética sugiere un nuevo intercambio interdependiente y abierto entre la dimensión conceptual y material del arte: al liberar la materialidad del objeto -y el discurso filosófico, las estructuras de poder y los paradigmas estéticos de la pura visualidad que lo rodean- esta noción ofrece el potencial predispuesto de continuar con la codificación conceptual, organización, distribución, contextualización e interpretación.

Interaction with materiality (even on screen), is part of the reason why art matters, both literally and figuratively. (Preez, 2017, p.67) 
La investigación concluye con una máquina digital, un algoritmo que decide por nosotros si el objeto que hemos producido pertenece a un sistema de fabricación digital o artesanal. Este dispositivo tiene por objeto plantear la siguiente pregunta:

¿Cómo pueden las máquinas revolucionar las ideas convencionales y los prejuicios de lo que debería ser la artesanía o la fabricación digital?

El machine learning, término que hace referencia a una disciplina de la informática relacionada con la inteligencia artificial (IA), sirve para crear sistemas que aprenden por sí solos. El software empleado para esta máquina es Teachable Machine, una plataforma de Google.

Trasladar a una IA el poder de decisión sobre la disciplina a la que pertenecen los objetos resultantes y las máquinas permite observar los conflictos a los que se enfrenta la máquina al encarar esta pregunta sempiterna. La máquina ofrece resultados en forma de porcentajes, por lo que puede ocurrir que decida que un objeto es totalmente (100\%) de una disciplina o situarlo en un estadio intermedio. Para enseñar al algoritmo a decidir, introduje previamente un banco de imágenes con objetos fabricados con las técnicas de producción propias de cada disciplina.

Alienarse y trasladar la agencia a la máquina a través de un algoritmo nos permite ver cómo se comporta ante objetos que se sitúan en ese umbral en el que estoy trabajando y al que quiero seguir trasladando preguntas.

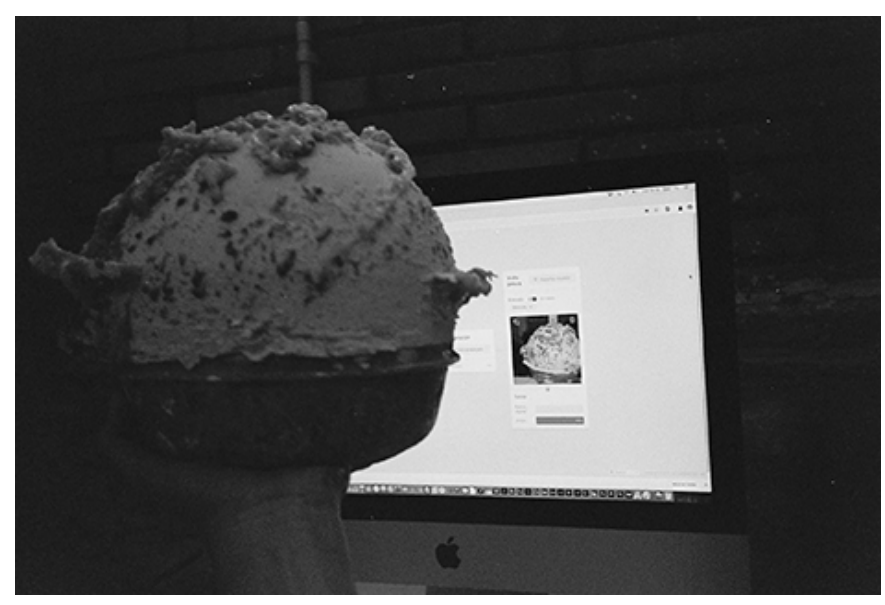

Figura 9. Teachable Machine 
Después de introducir en el algoritmo todas las piezas realizadas durante el proyecto, constaté que, cuanto más compleja es la forma que se observa, más se eleva el porcentaje de fabricación digital. La máquina entiende que la mano humana no es capaz de reproducir esas formas sin ayuda de un dispositivo o máquina. Cuanto más orgánica, natural y «sencilla» es la forma, más crece la proporción de artesanía. Los resultados que más se acercan a un punto intermedio, a ese umbral, son los de "la máquina de la bolita", pues son piezas de forma sencilla, circunferencias, pero hay cierta complejidad en el dibujo que componen.

\section{Conclusión. Algo que ha tenido lugar en mí}

En esta investigación se ha dado espacio al hacer y a la acción con el objetivo de abrir fronteras, entablar diálogos y negociar con la materia. También se ha dado espacio a la práctica como método para investigar en el diseño y desdibujar los límites de los ámbitos disciplinarios.

No puedo decir que no haya afectado a la investigación el contexto de la pandemia global en la que se ha desarrollado. Seguramente, en otras circunstancias, el proyecto sería algo distinto, aunque tampoco demasiado. Pero se trata de eso, de aceptar las circunstancias y adaptarse. El mundo cambia y nosotros con él, por lo que doy mucho valor a la honestidad con la que compartimos un proceso y a la necesidad de ser consecuentes con el mismo.

Este proyecto se explica en primera persona, pues se trata de un suceso concreto, de una experiencia propia, algo que ha tenido lugar en mí. He leído y transmitido la mirada que aportan otros autores y autoras sobre los temas que se tratan en la investigación, y otorgo un gran valor a lo que otras personas (del mundo académico o profesional) han podido observar. Pero también he errado, probado, preguntado, materializado, construido, documentado y experimentado de forma corpórea para poder aprender, dado que, como vengo defendiendo, considero que solo a través de la propia experiencia y del aprendizaje podemos construir nuestra propia mirada frente al mundo, así como la práctica que me ocupa.

Por eso presento las máquinas como una herramienta que activa experiencias, experiencias en las que el cuerpo y la materia piensan en conjunto y dejan espacio a lo aleatorio y lo casual, como puertas que 
abren otras oportunidades y otros caminos de experimentación que acaban generando nuevos aprendizajes.

Aprendizajes que, por suerte, considero provisionales, pues al ser compartidos con otros pueden crecer, transformarse y abrir nuevos caminos.

El proyecto pone el foco en los saberes tácitos, esos cuya principal característica de transmisión es que, sin la interacción directa y personal con aquello que los posee, resultan imposibles o, cuando menos, muy complejos. Por eso encuentro en el espacio del taller, en su más amplia concepción, un espacio de aprendizaje. Nos encontramos en un momento en el que la forma más tradicional de entender un taller, la de los maestros artesanos y sus herramientas, convive con los talleres de fabricación digital. Es el punto intermedio entre estas dos disciplinas el que me interesa y el que he querido explorar en esta investigación práctica: el umbral de incertidumbre que se genera al mezclar las técnicas, los lenguajes y sus formas de producción.

La relación entre los procesos artesanos y el diseño es ambigua, porque si bien es cierto que, por una parte, se toma como referencia el taller del artesano en la formación del diseñador, sus pretensiones ideológicas se sitúan más cerca del arte y su capacidad crítica.

Sin estos espacios corremos el riesgo de seguir entendiendo la investigación como un ciclo donde leer, escribir, resumir y transmitir a través de artículos se convierta en el único medio con el que aprender y transmitir el conocimiento. Aun siendo los artículos importantísimos para transmitir saberes, pues hacen posible y accesible su rápida difusión, quiero incidir aquí en que también es importante hacer.

Podemos, por ejemplo, leer cien libros sobre las propiedades del cemento y sus usos, pero hasta que no metamos la mano en la gaveta y comprobemos su densidad, no sabremos si la mezcla funciona. $\mathrm{O}$ como sucede a menudo, al copiar radicalmente la metodología de un referente y ponerla en práctica, vemos que hay muchos "trucos", experimentaciones o formas de resolver problemas durante el proceso que no nos explican, sobre todo en proyectos que involucran la materia, la técnica y las herramientas. Tendemos a enseñar "lo bonito" (el resul- 
tado) cuando lo interesante, donde el aprendizaje y la experiencia nos transforma, es el camino a recorrer (el proceso).

Camps y Rowan (2019) abogan que para validar estas prácticas son necesarios sistemas que no sean estrictamente cuantitativos ni cualitativos, quizás no demostrables ni objetivables, pero sí transferibles y sensibles a la calidad y la coherencia, a la materia, lo social, lo político, los cuerpos y los conceptos, y que necesariamente sitúen las prácticas de investigación en diseño en un marco de legitimidad y apertura.

Reflexiono sobre la manera en que se han pensado y valorado las comunidades de prácticas en relación con el mundo académico en el entorno del arte y el diseño en contraposición a las comunidades puramente académicas.

La comunidad académica está asociada a lo trascendente, lo científico, lo racional, lo universal y a investigaciones rigurosas y argumentadas con la "verdad". En cambio, a la comunidad de prácticas se le asocian adjetivos contrarios como, por ejemplo, intrascendente o mundano. Esto tiene que ver con la manera en la que históricamente se ha escrito, y hemos leído, la historia del arte desde el Renacimiento. En la que al saber-hacer mecánico se le habría otorgado la categoría de dimensión técnica, y se habrían rebajado los saberes tácitos que se producen en estos espacios más íntimos, malinterpretándolos junto con la cotidianidad de los conocimientos locales, desvalorizando así la alta carga cultural que poseen. Es frecuente que la crítica del arte descalifique una obra diciendo que es mera artesanía, lo que quiere decir que es puro virtuosismo vacío, carente de espíritu, o que se trata de una obra que no trasciende lo meramente local y costumbrista. Pero estamos volviendo a una época en la que el arte contemporáneo retoma la importancia de lo material y los procesos experimentales y deja a un lado el peso de la idea, lo conceptual. Ahora se habla de un periodo postconceptual.El proyecto termina dando voz y capacidad de decisión a una máquina, una inteligencia artificial que al no tener toda la información y al conocer solo los dos extremos de las disciplinas que se plantean (artesanía y fabricación digital), no puede posicionarse en el umbral del lenguaje y la técnica que propongo. Esto abre un debate que 
se desarrolla durante todo el proyecto: ¿qué puede aprender la máquina del humano? y viceversa ¿qué puede aprender el humano de la máquina? El proyecto se centra en pensar con la máquina, en generar una alianza entre lo humano, la materia y la máquina, un diálogo.

\section{Bibliografía}

Boserman, C.; Ricart, D., 2016. Metodologías de investigación materializadas. Entre maquetas, tostadoras, diagramas, rampas y cabinas. Inmaterial. Diseño, Arte y Sociedad. Vol. 1, n.o 1 (1). <https:// www.inmaterialdesign.com/index.php/mag/article/view/10>

Bryant, L., 2014. Onto-Cartography. Edinburgh: Edinburgh University Press.

Camps, M.; Rowan, J., 2019. Indisciplinares: explorando la práctica como método de investigación en diseño. Diseña, (14), 100-117. Doi: 10.7764/disena.14.100-117

Capella, J. and Vargas, R., 2011. Así nacen las cosas. Barcelona: Random House Mondadori. Dewey, J., 2004. Experiencia y Educación, Biblioteca Nueva (Madrid)

Eliasson, O., 2009. Los modelos son reales. Barcelona: Gustavo Gili.

Escobar, A., 2016. Autonomía y diseño, la realización de lo comunal. Popayán: Universidad del Cauca

Foucault, M., 1976. Vigilar y castigar: nacimiento de la prisión. Madrid: Siglo Veintiuno.

Gràffica.info, Cultura visual., 2019. Bruno Munari y los fundamentos del proceso del diseño. [en línea] Disponible en: https://graffica.info/ bruno-munari-y-los-fundamentos-del-proceso-del-diseno/ [Acceso18 Abr. 2019].

Haraway, D., 1995: Ciencia, cyborg y mujeres. La reinvención de la naturaleza, Madrid, Cátedra.

Heidegger, M., 2002. Was Heisst Denken?. Frankfurt am Main: Klostermann. (versión castellana: ¿Qué significa pensar?, Trotta Editorial, Madrid, 2010)

Lafuente, A. \& Alonso, A., 2017, Taller de prototipado. La hospitalidad como cultura y como tecnología, en: J. Pérez de Lama et al (eds.), Machines Loving Grace. Fabricación digital, arquitectura y buen vivir, Lugadero, Sevilla; pp. 32-37 
Morin, E., 2000. La mente bien ordenada: repensar la reforma, reformar el pensamiento. Barcelona: Seix Barral.

Munari, B.; Artal Rodríguez, C., 1983. ¿Cómo nacen los objetos?. Barcelona: G.Gili.

Openshaw, J.; Adamson, G., 2015. Postdigital Artisans: Craftsmanship With A New Aesthetic In Fashion, Art, Design And Architecture. Frame.

Pallasmaa, J., 2014. La mano que piensa. Barcelona: Gustavo Gili

Schouwenberg, L.; Bruinsma, M.; Heinrich, R., 2017. Material Utopias.

Amsterdam: Sandberg Instituut.

Sennett, R., 2008. El Artesano. Anagrama (Barcelona), Colección Argumentos.

Sztulwark, P., 2015. Componerse con el mundo. Modos del pensamiento proyectual. Buenos Aires: Editorial Nobuko, pp.39, 63.

Taylor; Francis. 2017. Cosmopolitical Encounters: Prototyping At The National Zoo In Santiago, Chile. [online] Disponible en: <https://www. tandfonline.com/doi/citedb$\mathrm{y} / 10.1080 / 17530350.2018 .1433705$ ? scroll=to- $\mathrm{p} \&$ needAccess $=$ true $>$ [Acceso 2 Abril 2020].
Ana Guerrero Ferro (Barcelona 1997), diseñadora transdisciplinar, Master en Inves- tigación y Experimentación en Diseño BAU. Actualmente participa de una beca de produc- ción en Sala d'Art Jove como parte del colectivo Espacio Hacer. 